\title{
Clinical utility gene card for McArdle disease
}

\author{
Rhonda L. Taylor ${ }^{1,2} \cdot$ Mark Davis $^{3} \cdot$ Emma Turner $^{1,2} \cdot$ Astrid Brull $^{4} \cdot$ Tomás Pinos $^{5,6} \cdot$ Macarena Cabrera $^{7} \cdot$ \\ Kristen J. Nowak ${ }^{2,8,9}$
}

Received: 2 May 2017 / Revised: 9 November 2017 / Accepted: 23 November 2017 / Published online: 25 January 2018

(c) European Society of Human Genetics 2018

\section{Name of the disease (synonyms)}

McArdle disease (glycogenosis type V; glycogen storage disease V (GSDV); PYGM deficiency; muscle glycogen phosphorylase deficiency; myophosphorylase deficiency).

\section{OMIM\# of the disease}

\#232600.

\section{Name of the analysed genes or DNA/chromosome segments}

Muscle glycogen phosphoryalse (PYGM).

\section{OMIM\# of the gene(s)}

$\# 608455$.

Review of the analytical and clinical validity as well as of the clinical utility of DNA-based testing for variants in the $P Y G M$ gene(s) in

$\triangle$ diagnostic,

$\triangle$ predictive and

$\triangle$ prenatal settings and for

$\otimes$ risk assessment in relatives.

Kristen J. Nowak

kristen.nowak@health.wa.gov.au

1 Centre for Medical Research, Faculty of Health and Medical Sciences, The University of Western Australia, Perth, WA, 6009, Australia

2 Harry Perkins Institute of Medical Research, QEII Medical Centre, QQ Block, Nedlands, WA 6009, Australia

3 Neurogenetics Laboratory, Department of Diagnostic Genomics, QEII Medical Centre, PP Block, Nedlands, WA 6009, Australia

4 Sorbonne Universités, UPMC Univ Paris 06, INSERM UMRS974, CNRS FRE3617, Center of Research in Myology, Paris 75013, France
5 Mitochondrial Pathology and Neuromuscular Disorders Laboratory, Vall d'Hebron Research Institute, Barcelona, Spain

6 Centro de Investigación Biomédica en Red de Enfermedades Raras, Barcelona, Spain

7 Neurology Department and Instituto de Biomedicina de Sevilla, Hospital Universitario Virgen del Rocío, Seville 41013, Spain

8 School of Biomedical Sciences, Faculty of Health and Medical Sciences, The University of Western Australia, Perth, WA 6009, Australia

9 Public and Aboriginal Health Division, Department of Health, Office of Population Health Genomics, East Perth, WA 6004, Australia 


\section{DISEASE CHARACTERISTICS}

\subsection{Name of the disease (synonyms)}

McArdle disease (glycogenosis type $\mathrm{V}$; glycogen storage disease V (GSDV); PYGM deficiency; muscle glycogen phosphorylase deficiency; myophosphorylase deficiency).

\subsection{OMIM\# of the disease}

\#232600.

\subsection{Name of the analysed genes or DNA/ chromosome segments}

Muscle glycogen phosphoryalse (PYGM).

\subsection{OMIM\# of the gene(s)}

\#608455.

\subsection{Mutational spectrum}

McArdle disease is the most common disorder of skeletal muscle carbohydrate metabolism. It is exclusively caused by recessive variants in the $P Y G M$ gene, which codes for the skeletal muscle glycogen phosphorylase (myophosphorylase) enzyme [1,2]. The myophosphorylase enzyme is the first enzyme in the process to breakdown glycogen stores in skeletal muscle.

Around 150 different disease-causing variants in PYGM have been reported with approximately half being missense variants, $\sim 18 \%$ deletions, $\sim 13 \%$ nonsense variants, $\sim 11 \%$ splice site variants and the remainder duplications or insertion/deletion variants $[1,3]$. The majority of variants are located within exons and all result in severely reduced or absent myophosphorylase enzyme activity, often as a result of nonsense-mediated decay [4].

Many variants are catalogued in the Leiden Open Variation Database (LOVD) (http://databases.lovd.nl/shared/va riants/PYGM).

\subsection{Analytical methods}

A diagnosis of McArdle disease is initially suspected based on patients self-reporting exercise intolerance, with cramps and myalgia [5]. Twenty-five per cent of patients develop fixed skeletal muscle weakness, which predominantly involves upper limbs [6,7]. Both ischaemic [8] and nonischaemic [9] forearm exercise tests can then be performed, where the absence of elevated pyruvate and lactate in venous blood from the contracting forearm is considered indicative of McArdle disease.
Basal creatine kinase (CK) levels in blood are usually elevated in McArdle patients. Exercise can precipitate further increases in CK levels and associated rhabdomyolysis episodes, which can lead to myoglobinuria and renal failure [10]. The 'second wind phenomenon' may also be present with marked improvement in tolerance to exercise and remission of tachycardia after $10 \mathrm{~min}$ of exercise [11]. Based on the above evidence, a diagnosis of McArdle disease is subsequently confirmed by histological analysis and/ or DNA sequencing of the PYGM gene. Subsarcolemmal and intermyofibrillar glycogen accumulation can be indicated by periodic acid Schiff staining; however, this method can also stain other polysaccharides. A lack of myophosphorylase by histochemistry is specific to McArdle disease [12].

The most efficient method of identifying the underlying disease-causing variant is to initially amplify the exon/s containing the most common variant in the ethnic group to which the patient belongs, and sequence the PCR product by traditional Sanger sequencing [1]. Alternatively, an approach that is becoming increasingly adopted is to sequence all 20 PYGM exons and flanking regions by massively parallel sequencing (MPS). In some instances, sequencing of patient RNA extracted from a skeletal muscle biopsy may be performed in order to confirm altered splicing.

\subsection{Analytical validation}

Variants identified by MPS methods can be validated by Sanger sequencing.

\subsection{Estimated frequency of the disease}

(Incidence at birth ('birth prevalence') or population prevalence. If known to be variable between ethnic groups, please report):

Based on affected individuals in national disease registries, estimated population prevalence varies from 1 in 100,000 individuals in the United States, to 1 in 140,000 individuals in Spain [7]. However, recent analyses of the allele frequencies of the two most common disease-causing variants in whole exome data from European Americans with no diagnosed skeletal muscle disease suggest that the actual prevalence may be closer to 1 in 50,000 [13]. This discrepancy is likely due to low rates of diagnoses, phenotypic heterogeneity and/or overestimation of disease penetrance [13].

Specific variants are enriched in certain ethnic groups, but there is little evidence to suggest that any one population may be more susceptible to McArdle disease than another. The most common variant in studied populations is the c.547C $>\mathrm{T} \quad$ (p.Arg50Ter) variant (NM_005609.2; 
NP_005600.1; exon 1), which occurs at a frequency of $\sim 30-80 \%$ in Caucasian populations (the Netherlands, $31 \%$; Italy, 43\%; Brazil, 50\%; Spain, 55\%; Germany, 58\%; USA, 60-63\%; France, 68-72\%; UK, 77-81\%) [1]. It is not known whether the p.Arg50Ter variant is due to a founder effect; however, in Asian populations this variant is rare. In Japanese populations, the most commonly occurring variant is c.2527_2529delTTC (p.Phe710del; NM_005609.2; NP_005600.1; exon 17), which accounts for $68 \%$ of cases [14].

\subsection{Diagnostic setting}

\begin{tabular}{lll} 
& Yes & No \\
A. (Differential) diagnosis & $\bigotimes$ & $\square$ \\
B. Predictive testing & $\bigotimes$ & $\square$ \\
C. Risk assessment in relatives & $\bigotimes$ & $\square$ \\
D. Prenatal & $\square$ & $\square$ \\
\hline
\end{tabular}

Comment: Some of the features of McArdle disease, including exercise intolerance, muscle pain, cramps and rhabdomyolysis, may be present in other neuromuscular disorders.

The most common differential diagnoses include other glycogen storage myopathies and lipid metabolism disorders. Among glycogen storage myopathies, type VII (Tarui disease; OMIM \#232800) caused by variants in the muscle phosphofructokinase gene (PFKM; OMIM \#61068) [15], type X (OMIM 261670) caused by variants in the muscle phosphoglycerate mutase gene (PGAMM; OMIM 612931), type XI (OMIM 612933) caused by variants in the lactate dehydrogenase A gene (LDHA; OMIM 150000), and phosphoglycerate kinase 1 (PGK1; OMIM 311800) deficiency (OMIM 300653) may also be considered.

Some lipid metabolism disorders can similarly produce clinical phenotypic overlap with McArdle disease, such as carnitine palmitoyl transferase II (CPT2; OMIM 600650) deficiency (OMIM 255110), very long-chain acyl-CoA dehydrogenase (ACADVL; OMIM 609575) deficiency (OMIM 201475) and less commonly mitochondrial trifunctional protein (HADHA; OMIM 600890, HADHB; OMIM 143450) deficiency (OMIM 609015). Variants in the phosphatidic acid phosphohydolase 1 gene (LPIN1) are the most common cause of rhabdomyolysis in children.

Other skeletal muscle conditions can less commonly present with exercise intolerance and rhabdomyolysis episodes, such us mitochondrial myopathies or muscular dystrophies. However, they are usually associated with other symptoms and signs that are not present in McArdle disease.
Definitive diagnoses are confirmed by identification of the disease-causing variants. Sequencing on a multi-gene panel, in most cases, resolves the differential diagnosis.

\section{TEST CHARACTERISTICS}

\subsection{Analytical sensitivity}

(proportion of positive tests if the genotype is present) Close to $100 \%$.

\subsection{Analytical specificity}

(proportion of negative tests if the genotype is not present) Close to $100 \%$.

\subsection{Clinical sensitivity}

(proportion of positive tests if the disease is present)

The clinical sensitivity can be dependent on variable factors such as age or family history. In such cases, a general statement should be given, even if a quantification can only be made case by case.

Due to phenotypic overlap with other diseases as outlined in Section 1.9, multiple genes can be considered for genetic screening if the clinical diagnosis is ambiguous. However, if an accurate clinical diagnosis has been made (based predominantly on forearm exercise test and/or presence of glycogen accumulation, and more specifically the absence of glycogen phosphorylase activity in a skeletal muscle biopsy), $100 \%$ of cases are expected to be positive for disease-causing variants in $P Y G M$.

\subsection{Clinical specificity}

(proportion of negative tests if the disease is not present)

The clinical specificity can be dependent on variable factors such as age or family history. In such cases, a general statement should be given, even if a quantification can only be made case by case.

Clinical specificity is expected to be high, although asymptomatic individuals are not routinely tested and therefore a precise proportion cannot be ascertained. However, there are no individuals that are homozygous for nonsense, frameshift or splice site variants present in the ExAC database (http://exac.broadinstitute.org) of individuals of non-paediatric disease, suggesting full penetrance of the disease.

\subsection{Positive clinical predictive value}

(life time risk to develop the disease if the test is positive) 
Close to $100 \%$. Milder phenotypes have been observed for patients harbouring intronic splice site variants in combination with p.Arg50Ter or c.1112G >A (p.Gly205Ser; NM_005609.2; NP_005600.1; exon 5) [16]. As little as $2-5 \%$ of myophosphorylase activity may be sufficient to prevent disease occurrence or progression such that some variants that allow 'leaky' transcription of a functional copy of the gene may not progress to disease manifestation [16].

\subsection{Negative clinical predictive value}

(Probability not to develop the disease if the test is negative)

Assume an increased risk based on family history for a non-affected person. Allelic and locus heterogeneity may need to be considered.

Index case in that family had been tested: Negative clinical predictive value is close to $100 \%$.

Index case in that family had not been tested: Close to 100\%; however, genetic testing would usually not be undertaken in clinically unaffected individuals.

\section{CLINICAL UTILITY}

\section{1 (Differential) diagnostics: The tested person is clinically affected}

(To be answered if in 1.9 'A' was marked)

\subsubsection{Can a diagnosis be made other than through a genetic test?}

No $\otimes$ (continue with

$$
\text { 3.1.4) }
$$

Yes $\square$

$\begin{array}{ll}\text { Clinically } & \square \\ \text { Imaging } & \square \\ \text { Endoscopy } & \square \\ \text { Biochemistry } & \square \\ \text { Electrophysiology } & \square\end{array}$

Other (please describe) $\otimes$ Histological and histochemical analysis of skeletal muscle biopsies. The myophosphorylase enzyme is the first enzyme in the process to breakdown glycogen stores in skeletal muscle. Therefore, an absence of myophosphorylase (combined with an accumulation of glycogen) might
Table (continued)

be considered diagnostic, although it is not a definitive diagnosis.

\subsubsection{Describe the burden of alternative diagnostic methods to the patient}

A genetic test with identification of homozygous or compound heterozygous variants in the $P Y G M$ gene is required in order to make a definitive diagnosis of McArdle disease. However, a number of other phenotypic tests are generally performed prior to genetic screening. These include the forearm test, measurement of the second wind phenomena by treadmill excercise, and skeletal muscle biopsy [7].

Since McArdle disease causes skeletal muscle pain and damage, exercise-based tests often result in physical discomfort. Open skeletal muscle biopsy can also be considered invasive and may lead to significant scarring, however percutaneous needle biopsy can also be performed which reduces the negative impact on patients [17].

\subsubsection{How is the cost effectiveness of alternative diagnostic methods to be judged?}

As genetic testing is required in order to provide a definitive diagnosis, complementary tests indicating diagnosis are additional rather than alternative costs. Targeted screening of specific exons by Sanger sequencing based on those commonly mutated in the ethnic groups of the patient may be the most cost-effective approach [1] to genetic testing (Section 1.6). However, as the cost of MPS continues to lessen, a gene panel or whole exome/genome sequencing approach is a cheaper option than Sanger sequencing all 20 exons if no variant is identified in the exon harbouring the most prevalent variant in the patient's population.

3.1.4 Will disease management be influenced by the result of a genetic test?

No $\square$
Yes $\square$

Therapy (please No curative therapy presently describe) exists, but physical training improves symptoms [7]. Many putative treatments to restore phosphorylase activity have been tested, including viral delivery of myophosphorylase cDNA [18] and 
Table (continued)

a range of pharmaceutical agents

(e.g. aminoglycosides $[19,20]$ and histone deacetylase inhibitors

[21]).

Prognosis (please Due to a poor genotype-phenotype describe)

Management (please describe) correlation, a genetic diagnosis will not inform about disease progression but can influence the decision to implement lifestyle changes before serious disease complications manifest. For example, the clinical course of McArdle disease can be improved with exercise conditioning [7].

High intensity exercise (e.g. lifting weights and sprinting) and any exercise causing pain should be avoided due to the occurrence of skeletal muscle damage. However, aerobic training programmes with adequate supervision can improve exercise tolerance in McArdle disease patients [5]. The amount and type of exercise that precipitates symptoms among patients varies, though isometric exercise and sustained dynamic exercise are likely to cause problems [15]. Rhabdomyolysis can lead to a lifethreatening situation. It requires immediate intervention in order to prevent renal failure and correct metabolic disturbances if present. The ingestion of sugars immediately prior to exercise [22] and adoption of a carbohydrate-rich diet have been found to improve exercise tolerance, thus potentially mitigating exercise-induced rhabdomyolysis.

Converting to a diet rich in complex carbohydrates to facilitate a ready supply of blood glucose is a beneficial long-term intervention for McArdle disease patients [23].
3.2 Predictive setting: The tested person is clinically unaffected but carries an increased risk based on family history

(To be answered if in 1.9 'B' was marked).

\subsubsection{Will the result of a genetic test influence lifestyle and prevention?}

If the test result is positive (please describe).

Lifestyle modifications that have a positive influence on disease phenotype as already indicated (e.g. a short warm up period prior to exercise to evoke a second wind, a complex carbohydrate-rich diet, and exercise training) can be commenced.

If the test result is negative (please describe)

Introducing lifestyle modifications that modulate the disease phenotype are then not necessary.

\subsubsection{Which options in view of lifestyle and prevention does a person at-risk have if no genetic test has been done (please describe)?}

The lifestyle modifications as indicated for a person receiving a positive molecular diagnosis.

\subsection{Genetic risk assessment in family members of a diseased person}

(To be answered if in 1.9 'C' was marked).

\subsubsection{Does the result of a genetic test resolve the genetic situation in that family?}

Almost always. The disease severity varies vastly between patients, even those from the same family [24]. The typical age of onset can range from early or late childhood through to adulthood, and some patients exhibit few symptoms. Whilst for others, their disease can be severely incapacitating. For severely affected patients, even mild activities, such as brushing teeth or chewing gum, can cause skeletal muscle pain, weakness and stiffness. Therefore, in many instances, a molecular diagnosis for one family member is highly indicative for other family members exhibiting a skeletal muscle disease phenotype. However, differences in severity may complicate the situation in some families.

\subsubsection{Can a genetic test in the index patient save genetic or} other tests in family members?

Yes, a genetic test can save other tests. Genetic testing should still be performed to confirm diagnosis, but other procedures that usually assist in making the diagnosis (e.g. 
skeletal muscle biopsy) may not be necessary for individuals with characteristic clinical symptoms who also have a family member diagnosed with the disease. However, as mentioned in the previous section, phenotype severity can vary greatly even within the same family.

\subsubsection{Does a positive genetic test result in the index patient enable a predictive test in a family member?}

Yes. Performing predictive genetic testing for at-risk family members before the advent of symptoms is recommended as knowledge of a positive test result can facilitate the early implementation of the recommended dietary and exercise changes for maximum benefit. These in turn can lessen disease severity and adverse effects such as kidney damage.

\subsection{Prenatal diagnosis}

(To be answered if in 1.9 'D' was marked).

\subsubsection{Does a positive genetic test result in the index patient enable a prenatal diagnosis?}

Yes, although prenatal testing for McArdle disease is thought to be extremely rare due to the possible positive impact on disease phenotype from appropriate lifestyle measures. If the parents of a McArdle disease patient were to have a subsequent pregnancy (and both parents were proven carriers of a disease-causing $P Y G M$ variant), there would be a $25 \%$ chance of the foetus also being affected. All offspring from a McArdle disease patient would typically be heterozygous for a $P Y G M$ variant (carriers of McArdle disease), unless the other parent was also a carrier or a McArdle disease patient. In these scenarios, there would be a 50 or $100 \%$ likelihood, respectively, that the resulting child would have McArdle disease.

\section{IF APPLICABLE, FURTHER CONSEQUENCES OF TESTING}

Please assume that the result of a genetic test has no immediate medical consequences. Is there any evidence that a genetic test is nevertheless useful for the patient or his/her relatives? (Please describe).

Genetic testing can have a positive impact on patient and family well-being by providing greater understanding of the cause of disease. Further, patients are able to implement lifestyle changes that relieve symptoms and improve long-term outcomes. While no curative treatment presently exists, a genetic diagnosis may become essential for future therapies that target the underlying molecular defect.
Acknowledgements This work was supported by EuroGentest2 (Unit 2: 'Genetic testing as part of health care'), a Coordination Action under FP7 (Grant Agreement Number 261469) and the European Society of Human Genetics. KJN was funded by an Australian Research Council Future Fellowship (FT100100734), and MC by Instituto de Salud Carlos III (JR15/00042).

\section{Compliance with ethical standards}

Conflict of interest The authors declare that they have no conflict of interest.

\section{References}

1. Nogales-Gadea G, Brull A, Santalla A, Andreu AL, Arenas J, Martín MA, et al. McArdle disease: update of reported mutations and polymorphisms in the PYGM gene. Hum Mutat. 2015;36:669-78.

2. Gautron S, Daegelen D, Mennecier F, Dubocq D, Kahn A, Dreyfus J-C. Molecular mechanisms of McArdle's disease (muscle glycogen phosphorylase deficiency). J Clin Invest. 1987;79:275-81.

3. Vieitez I, Teijeira S, Fernandez JM, San Millan B, Miranda S, Ortolano S, et al. Molecular and clinical study of McArdle's disease in a cohort of 123 European patients. Identification of 20 novel mutations. Neuromuscul Disord. 2011;21:817-23.

4. Nogales-Gadea G, Rubio JC, Fernandez-Cadenas I, GarciaConsuegra I, Lucia A, Cabello A, et al. Expression of the muscle glycogen phosphorylase gene in patients with McArdle disease: the role of nonsense-mediated mRNA decay. Hum Mutat. 2008;29:277-83.

5. Lucia A, Nogales-Gadea G, Pérez M, Martín MA, Andreu AL, Arenas J. McArdle disease: what do neurologists need to know? Nat Clin Pract Neurol. 2008;4:568-77.

6. Nadaj-Pakleza AA, Vincitorio CM, Laforêt P, Eymard B, Dion E, Teijeira S, et al. Permanent muscle weakness in McArdle disease. Muscle Nerve. 2009;40:350-7.

7. Lucia A, Ruiz JR, Santalla A, Nogales-Gadea G, Rubio JC, García-Consuegra I, et al. Genotypic and phenotypic features of McArdle disease: insights from the Spanish national registry. J Neurol Neurosurg Psychiatry. 2012;83:322-8.

8. Zaman Z, De Raedt S. Ischemic exercise testing in suspected McArdle disease. Clin Chem. 2000;46:1198-9.

9. Kazemi-Esfarjani P, Skomorowska E, Jensen TD, Haller RG, Vissing J. A nonischemic forearm exercise test for McArdle disease. Ann Neurol. 2002;52:153-9.

10. Maté-Muñoz JL, Moran M, Pérez M, Chamorro-Viña C, GómezGallego F, Santiago C, et al. Favorable responses to acute and chronic exercise in McArdle patients. Clin J Sport Med. 2007; 17:297-303.

11. Vissing J, Haller RG. A diagnostic cycle test for McArdle's disease. Ann Neurol. 2003;54:539-42.

12. Dubowitz V, Sewry CA. Muscle biopsy: a practical approach. 3rd ed. Oxford: Elsevier; 2013.

13. De Castro M, Johnston J, Biesecker L. Determining the prevalence of McArdle disease from gene frequency by analysis of next-generation sequencing data. Genet Med. 2015;17:1002-6.

14. Sugie H, Sugie Y, Ito M, Fukuda T, Nonaka I, Igarashi Y. Genetic analysis of Japanese patients with myophosphorylase deficiency (McArdle's disease): single-codon deletion in exon 17 is the predominant mutation. Clin Chim Acta. 1995;236:81-6.

15. Di Mauro S. Muscle glycogenoses: an overview. Acta Myol. 2007;26:35-41. 
16. Vissing J, Duno M, Schwartz M, Haller RG. Splice mutations preserve myophosphorylase activity that ameliorates the phenotype in McArdle disease. Brain. 2009;132:1545-52.

17. Bergstrom J. Percutaneous needle biopsy of skeletal muscle in physiological and clinical research. Scand J Clin Lab Invest. 1975;35:609-16.

18. Howell JM, Walker KR, Davies L, Dunton E, Everaardt A, Laing N, et al. Adenovirus and adeno-associated virus-mediated delivery of human myophosphorylase cDNA and LacZ cDNA to muscle in the ovine model of McArdle's disease: Expression and re-expression of glycogen phosphorylase. Neuromuscul Disord. 2008;18:248-58.

19. Schroers A, Kley R, Stachon A, Horvath R, Lochmuller H, Zange $\mathrm{J}$, et al. Gentamicin treatment in McArdle disease: failure to correct myophosphorylase deficiency. Neurology. 2006;66:285-6.

20. Birch KE, Quinlivan RM, Morris GE. Cell models for McArdle disease and aminoglycoside-induced read-through of a premature termination codon. Neuromuscul Disord. 2013;23:43-51.
21. de Luna N, Brull A, Guiu JM, Lucia A, Martin MA, Arenas J, et al. Sodium valproate increases the brain isoform of glycogen phosphorylase: looking for a compensation mechanism in McArdle disease using a mouse primary skeletal-muscle culture in vitro. Dis Model Mech. 2015;8:467-72.

22. Vissing J, Haller RG. The effect of oral sucrose on exercise tolerance in patients with McArdle's disease. N Engl J Med. 2003;349:2503-9.

23. Andersen ST, Vissing J. Carbohydrate- and protein-rich diets in McArdle disease: effects on exercise capacity. J Neurol Neurosurg Psychiatry. 2008;79:1359-63.

24. Paradas C, Fernandez-Cadenas I, Gallardo E, Lligé D, Arenas J, Illa I, et al. Variable presentation of the clinical phenotype of McArdle's disease in a kindred harbouring a novel compound genotype in the muscle glycogen phosphorylase gene. Neurosci Lett. 2005;391:28-31. 